\title{
LA ACTIVIDAD COMERCIAL EN EMPRESAS TURÍSTICAS. PERCEPCIÓN DE LOS ESTUDIANTES DE TURISMO
}

\author{
Pedro Canales Ronda \\ Asunción Hernández Fernández. \\ Universitat de València
}

\section{RESUMEN}

El presente trabajo pretende analizar la opinión que los estudiantes universitarios de Turismo tienen respecto a desarrollar su futuro laboral en el área comercial de empresas turísticas, la que más oferta de puestos de trabajo genera en España. En concreto, se pretende conocer cuál es su percepción sobre este tipo de trabajo, tanto desde un punto de vista personal como profesional. Para alcanzar estos objetivos, se ha encuestado a 348 estudiantes de diferentes cursos, de grado y posgrado, que han sido clasificados en función de su interés por trabajar como vendedores en el sector turístico. Los resultados muestran que, en general, la valoración de una futura actividad de tipo comercial es positiva.

Palabras clave: Gestión comercial, percepción, imagen del vendedor, orientación al cliente, ética.

\section{The commercial activity of tourism companies. Perception of tourism students}

\section{ABSTRACT}

The current research intends to analyze the opinion that the tourism university students have regarding to develop their future labor in the commercial management area of tourist companies, the one that more offer of jobs generates in Spain. Specifically, we want to know what the perception of this type of work is, both from a personal and professional point of view. To achieve these objectives, 348 students, from different undergraduate and postgra-

Fecha de recepción: 12 de diciembre de 2017

Fecha de aceptación: 22 de mayo de 2018

Departamento de Comercialización e Investigación de Mercados. Facultad de Economía. Universitat de València. Avda. de los Naranjos s/n. 46022 VALENCIA (España). E-mail: pedro.canales@uv.es, asunción.hernandez@uv.es 
duate courses, have been surveyed, who have been classified according to their interest in working as sellers in the tourism sector. The results show that, in general, the valuation of a future commercial activity is positive.

Keywords: Commercial management, perception, image of the salesperson, customer orientation, ethics.

\section{INTRODUCCIÓN}

Las tareas relacionadas con la gestión comercial, especialmente la venta personal, son la actividad de marketing que más recursos necesita y genera en las empresas (Barat y Spillan, 2009; Concha, Kumar, Tarail y Wilson, 2014). No obstante, y desde la visión de la sociedad, el trabajo de vendedor, comercial (o nombres similares), no goza de suficiente reconocimiento y prestigio como para ser considerado una verdadera profesión (Honeycutt, Ford, Swenson y Swinyard, 1999; Wiles y Spiro, 2004; Waldeck, Pullins y Houlette, 2010; Bahhouth, Spillan y Karsakalian, 2014). Incluso algunos autores (Hartman, 2006) han analizado el efecto negativo que sobre esta actividad profesional han tenido algunas películas, y otro tipo de ficciones. Generalmente han presentado a los vendedores con estereotipos negativos, más relacionados con el vendedor puerta a puerta, que con el gestor comercial necesario en cualquier actividad que desee alcanzar el éxito. Quizá por ello, muchas personas con formación universitaria no se plantean desarrollar su carrera profesional en el entorno de la gestión comercial y las ventas (Manning, Reece y Ahearne, 2010; Ballestra, Cardinali y Palanga, 2017). Esta visión negativa también se puede observar en algunos medios de comunicación, por tanto como reflejo de la supuesta realidad (Lee, Sandfield y Dhaliwal, 2007).

Por otro lado, en entornos cada vez más competitivos y con unos clientes cada vez más informados y exigentes, se está produciendo un cambio en las estructuras comerciales de las empresas, en concreto en el sector turístico, lo que las obliga a estar mucho más centradas en el cliente, dejando de lado la preocupación sólo por su oferta. En este nuevo contexto, la función comercial está experimentando importantes cambios, pasando de una función empresarial aislada y puramente de acción, a una función más estratégica y en constante relación con el resto de la empresa (Ingram, LaForge y Leigh, 2002; Storbacka, Ryals, Davies y Nenonen, 2009; Keszey y Biemasn, 2017). Además, esta nueva complejidad está afectando a la venta personal más que en cualquier otra área funcional de las empresas (Honeycutt, Ford, Swenson y Swinyard, 1999). Por ello, en el entorno empresarial competitivo de hoy en día, la venta requiere altos niveles de profesionalidad, de capacidades organizativas y de gestión, así como de los conocimientos necesarios para que la gestión comercial se parezca a la de los consultores (Cron, Baldauf, Leigh y Grossenbacher, 2014). Los vendedores se han convertido tanto en gestores de relaciones como en creadores de valor para los clientes actuales y futuros (Weitz y Bradford , 1999; Blocker, Cannon, Panagopoulos y Sager, 2012), cambios que no son ajenos al sector turístico (Lillo, Ramón y Sevilla, 2007; Lillo, 2009). Por todas estas razones, las empresas turísticas 
actuales necesitan vendedores que sean diferentes a los del pasado; necesitan personas brillantes, motivadas, formadas y que tengan las habilidades necesarias para adaptarse a un entorno empresarial en constante cambio (Pettijohn y Pettijohn, 2009).

Para satisfacer esta necesidad de vendedores cualificados, las empresas están recurriendo cada vez más a la captación de personas con formación universitaria (Wiles y Spiro, 2004). Los estudiantes universitarios son candidatos interesantes porque suelen ser jóvenes, inteligentes y con capacidad para aprender (Caballero y Walker, 2010) y pueden tener éxito en el actual panorama de la función comercial en las empresas (Peltier, Cummins, Pomirleanu, Cross y Simon, 2014). Por ello, algunas universidades, además de los contenidos en las asignaturas de marketing, han desarrollado programas específicos de formación relacionados con la gestión comercial y las ventas en el sector turístico. Esta formación permiten a sus alumnos adquirir los conocimientos y habilidades necesarios para triunfar en esta área profesional (Bolander, Bonney y Satornino, 2014).

No obstante, los estudiantes universitarios aún parecen tener una imagen no demasiado positiva del trabajo de ventas, debido a múltiples influencias de su entorno (Bahhouth, Spillan y Jözeowski, 2011); por ejemplo, respecto a la imagen ética de los vendedores y sus actuaciones profesionales (Burnett, Pettijohn y Keith, 2008; Castleberry, 2014) o los estereotipos inexactos sobre la naturaleza de las ventas (Cummins, Peltier, Pomirleanu, Cross y Simon, 2015). Por ello es menos probable que consideren desarrollar su futuro profesional en actividades de tipo comercial.

En el caso concreto de los estudiantes universitarios de turismo, el libro blanco "Título de grado en Turismo" (2004) editado por la Agencia Nacional de la Evaluación de la Calidad y Acreditación (ANECA) recoge, entre las múltiples figuras profesionales del turismo, diversas de estas relacionadas directamente con la función comercial (por ejemplo, director comercial de hotel o de touroperador). También, en el perfil profesional de otras figuras, indica el desarrollo de actividades comerciales, por ejemplo, director de hotel. Por su parte, el "Libro Blanco de los Recursos Humanos del Turismo" (2006) elaborado por Exceltur, reconoce la importancia de la formación en cuestiones comerciales para determinados puestos de trabajo.

Además, es de destacar la importancia cuantitativa que está teniendo en los últimos años la incorporación de personal con formación universitaria en el sector turístico (Marrero, 2015).

Tras esta introducción, los objetivos del presente estudio se centran en conocer la opinión de los estudiantes universitarios de turismo respecto a las tareas de la gestión comercial de las empresas turísticas y sus expectativas profesionales en la misma. En el siguiente epígrafe realizamos una revisión de la literatura que analiza este tema, para posteriormente plantear el desarrollo de nuestra investigación, analizar los resultados de la misma y finalizar con conclusiones e implicaciones formativas universitarias.

\section{REVISIÓN DE LA LITERATURA}

Las investigaciones llevadas a cabo por diferentes autores respecto a la valoración por parte de los estudiantes universitarios del trabajo de los vendedores, así como su interés en desarrollar su carrera profesional en el área de la venta personal, han sido en algunos 
casos contradictorios. En un estudio intercultural, analizando las percepciones de los estudiantes de diversos países americanos, respecto del trabajo de los vendedores y su interés por seguir una carrera profesional en ventas (Barat y Spillan, 2009) se determinó una percepción negativa. Sin embargo, otros estudios realizados unos años antes, revelaron que las percepciones de los estudiantes universitarios eran, en general, positivas (Dubinsky, 1980; Muehling y Weeks, 1988; Amin, Hayajneh y Nwakanma, 1995).

\subsection{Percepción de la actividad comercial en el sector turístico}

La revisión realizada de la literatura nos ha permitido conocer que, en la gran mayoría de los estudios realizados, el interés de los estudiantes universitarios respecto a seguir una carrera profesional en ventas no es muy elevado y suele mostrarse una visión negativa de dicha profesión como una carrera profesional futura (Dubinsky, 1980; Honeycutt, Ford, Swenson y Swinyard, 1999; Wiles y Spiro, 2004; Caballero y Joonas, 2009; Waldeck, Pullins y Houlett, 2010; Canales, 2012; Agnihotril, Bonney, Dixon, Erffemeyer, Pullins, Sojka y West, 2014). La posible explicación a esta percepción negativa puede deberse a la imagen del vendedor que presiona a sus potenciales clientes para lograr más ventas que sus competidores en un entorno competitivo muy agresivo y en el que la oferta excedía la demanda (Kerin, Hartley y Rudelius, 2009).

Incluso, otras investigaciones indican que las ventas aún no llegan a ser reconocidas como profesión (Hawes, Rich y Widmier, 2004; Waldeck, Pullins y Houlette, 2010). La actividad de gestión comercial y ventas todavía es considerada como profesión de escaso prestigio (Bristow, Amyx, Castleberry y Cochacran, 2011), poco o nada ética (Klein, Laczniak y Murphy, 2006; Pettijohn, Pettijohn y Taylor, 2007; Lee, Beatson, Garrett, Lings y Zhang, 2009, Mossaz y Coghlan, 2017) o para la que es necesario no tener muchos escrúpulos (Kavas, 2003).

Estos estereotipos negativos no están restringidos a un único entorno sociocultural. Estudios realizados en diferentes entornos y países, encontraron que la venta no es considerada entre los universitarios como una carrera profesional atractiva (Lee, Sandfield y Dhaliwal, 2007; Barat y Spillan, 2009; Canales, 2012; Agnihotril, Bonney, Dixon, Erffemeyer, Pullins, Sojka y West, 2014), algo que ocurre incluso en países en vías de desarrollo como Ghana (Bahhouth, Spillan y Mensah, 2013).

No obstante, y pese a estas percepciones no muy positivas, la actividad comercial y de ventas es un elemento fundamental de las estrategias de marketing en las empresas turísticas. Se trata de una elemento clave en la generación de recursos financieros necesarios para alcanzar el éxito y reconocimiento empresarial (Barat y Spillan, 2009; Concha, Kumar, Tarasil y Wilson, 2014; Paul y Shrivastava, 2015), y esto sólo puede lograrse con el personal de ventas adecuado. Por tanto, los responsables de los departamentos comerciales deben ser conscientes de la necesidad de aportar un valor añadido a las relaciones que mantienen con sus clientes y centrar el marketing en estos (Bristow y Gulati, 2002; Oviedo-García, 2007; Marcos-Cuevas, Critten, Squire y Speakman, 2014). Así, las empresas turísticas deben focalizar su fuerza comercial hacia las relaciones con el cliente, haciendo hincapié en una red comercial formada por personas flexibles, innovadoras, motivadas por el trabajo en sí mismo, con control emocional (Oviedo-Garcia, 
2007; Bagozzi, Berlschak y Verbeke, 2010; Lassk, Ingram, Kraus y Dimascio, 2012) y que estén familiarizados con las nuevas tecnologías (Bristow y Gulati, 2002; Walker, Tsarenko, Wagstaff, Powell, Steel y Brace-Govan, 2009; Concha, Kumar, Tarasil y Wilson, 2014).

Por tanto, la tradicional visión de los vendedores que sólo utilizan características y condiciones de su oferta para intentar convencer al cliente y lograr el cierre de la venta, está desfasada. Hoy en día, una fuerza comercial de éxito utiliza de forma activa las propuestas de valor para el cliente a fin de mantenerlos satisfechos y fieles a su empresa (Blocker, Cannon, Panagopoulos y Sager, 2012). Si bien no parece existir una única definición de venta orientada al cliente, todas centran su enfoque en satisfacer sus necesidades. Desarrollando relaciones duraderas con los mismos (Schwepker, 2003) como parte de la cultura de la organización comercial (Cross, Brasher, Rigdon y Bellenger, 2007).

Además, la orientación al cliente debe ser entendida y aplicada como un elemento motivador del equipo comercial. Mejora el compromiso laboral de los vendedores, tanto desde un punto de vista de la motivación extrínseca como intrínseca. Por ejemplo, ayuda a alcanzar los objetivos y facilitar el cumplimiento de las normas, y permite el logro de los objetivos de sus clientes (Bakker y Demerouti, 2007). Dado que los vendedores trabajan en un entorno fronterizo entre los clientes y su empresa, esta orientación y su efecto motivador, permiten que el trabajo sea percibido como más gratificante.

\subsection{El comportamiento ético en la gestión comercial turística}

El comportamiento ético se refiere a las percepciones del vendedor sobre los estándares éticos que se reflejan en las prácticas, los procedimientos, las normas y los valores de la organización para la que trabaja (Babin, Boles y Robin 2000; Schwepker 2001). La ética comercial (McClaren, 2000) sugiere que la percepción de un vendedor de los problemas éticos, y las alternativas de respuesta, está influenciada por varios factores, entre ellos, las normas morales aceptadas socialmente, los códigos de conducta de las empresas, la probabilidad de consecuencias positivas o negativas y el sesgo de juicio individual en las evaluaciones de situaciones éticas.

Para hacer frente a posibles situaciones no deseadas, las empresas han desarrollado procesos de mejora en la toma de decisiones éticas. Algunas empresas han diseñado códigos de conducta ética empresarial, que asocian a parte de sus incentivos y desincentivos, y tratan de influir en el pensamiento ético de cada uno de sus vendedores individuales. Estas pautas organizativas reducen el riesgo del vendedor de incumplir lo que su cliente, y la empresa para la que trabaja, esperan. Actuando de esta forma el vendedor genera una sensación personal de confort y seguridad (Babin, Boles y Robin 2000, Valentine y Barnett, 2003). También, cuando los clientes creen que un vendedor trabaja para una empresa ética, es probable que vean al vendedor como ético y creíble, lo que les facilitará la promoción de sus productos y el desarrollo de relaciones de fidelidad a largo plazo con los clientes (Wright y Lundstrom 2004).

Desde un punto de vista ético, la gente no suele tener una buena imagen de los vendedores. Esta mala imagen ha repercutido tradicionalmente en un escaso interés por desarrollar esta profesión. En el caso de los estudiantes universitarios la mala imagen limita sus 
salidas profesionales, pese a que los beneficios económicos de trabajar en ventas pueden ser elevados (Luthy, 2007; Ramsey, Marshall Johnston y Deeter-Schmelz, 2007).

Una de las posibles explicaciones a este comportamiento lo podemos encontrar en el entorno laboral del vendedor, que suele trabajar en solitario y debe satisfacer tanto a sus clientes como a la empresa para la que trabaja, y esto no siempre es fácil (Wortruba, 1996). Sin embargo, también hay una mayor conciencia de que las conductas poco éticas, incluso cuando son legales, pueden dañar la imagen y reputación del vendedor y de la empresa a la que representa. Este comportamiento no ético puede acabar provocando que el cliente deje de serlo, que se reduzca la fuerza moral de los empleados y se incremente la rotación de los vendedores (Thomas, Schermerhorn y Dienhart 2004).

Pero, no solo los vendedores, también los responsables de la gestión comercial están sujetos a intensas presiones internas y externas, que pueden marginar la preocupación por un adecuado comportamiento ético (Schwepker y Good, 2004). Como resultado, pueden surgir confusiones operativas respecto a las directivas éticas, y un aumento de la probabilidad de que los vendedores desarrollen comportamiento contrarios a la ética; además, altos estándares éticos son vitales para construir relaciones a largo plazo y de lealtad con los clientes (Mulki, Jaramillo y Locander, 2009).

\subsection{Objetivos de la investigación}

Tras la revisión de la literatura sobre el tema, y en base a la importancia de la gestión comercial como futuro profesional de los estudiantes universitarios de turismo, el objetivo general del presente estudio se centra en conocer la opinión de los estudiantes universitarios de turismo sobre el trabajo comercial. En concreto podemos indicar como principales objetivos los siguientes:

- Conocer cómo perciben los estudiantes de turismo las tareas de gestión comercial como una posible salida profesional tras acabar sus estudios universitarios.

- Analizar la visión que, desde un punto del comportamiento en el desarrollo de su actividad profesional, tienen los estudiantes universitarios de turismo del comportamiento de los vendedores.

- Conocer la opinión de los estudiantes sobre determinadas pautas de comportamiento no ético por parte de los vendedores en el desarrollo de la actividad comercial.

\section{METODOLOGÍA DE LA INVESTIGACIÓN}

Para alcanzar los objetivos planteados con este estudio se procedió a adaptar cuestionarios utilizados previamente en estudios similares (Dabholkar y Kellaris, 1992; Bristow, Amyx y Slanck, 2006). Para la versión definitiva del cuestionario (Cravens, Ingram, LaForge y Young., 1993; Ruiz, 1996) se consideraron las opiniones de expertos en la materia, tanto profesionales como académicos. También se tuvo en cuenta la opinión de un grupo de estudiantes a los que se les realizó un pretest del cuestionario inicial con el objetivo de analizar tanto aspectos semánticos como aspectos técnicos y la duración del 
mismo; tras este proceso se realizaron algunas modificaciones para mejorar la comprensión y la utilidad del mismo.

El cuestionario definitivo estaba formado por dos grupos de cuestiones. En la primera parte se solicitaba la opinión de los alumnos de turismo sobre el trabajo de los comerciales; en concreto se les pedía su opinión acerca de diversos aspectos del trabajo comercial (16 ítems) y sobre el comportamiento personal relacionado con el mundo de las tareas comerciales en el sector turístico (20 ítems). En todos los casos, se utilizó una escala tipo Likert de 5 puntos ( 1 = "Muy en desacuerdo -Totalmente inaceptable" hasta 5="Muy de acuerdo - Totalmente aceptable"). En la segunda parte se les pedía información sobre su relación actual y futura con las tareas profesionales del entorno comercial, así como su situación laboral del presente. Por último, se contemplaron preguntas de clasificación del encuestado (género, edad y curso de los estudios universitarios de turismo).

Los participantes en el estudio fueron estudiantes universitarios, que se hallaban cursando estudios universitarios de Turismo (grado o máster) en una universidad española, los cuales de modo voluntario contestaron, durante una de las sesiones, a las preguntas que el cuestionario les planteaba. El total de cuestionarios que se consideraron válidos, tras un proceso de revisión, fue 348. Respecto al perfil demográfico de los encuestados podemos indicar que, la media de edad de los participantes en el estudio es de 22,6 años, con una moda de 21; y el grupo mayoritario lo forman las mujeres $(53,7 \%)$. La distribución por cursos es la siguiente: primer curso 5,9\%, segundo curso $11,5 \%$, tercer curso 54,0\%, cuarto curso $20,9 \%$ y máster $7,7 \%$. Respecto al dato relativo a haber cursado alguna asignatura relacionada con la gestión comercial-ventas, el 84,7\% sí que lo han hecho. Dado que son estudiantes, la mayoría de los encuestados no trabajan o lo hacen ocasionalmente.

\subsection{Técnicas de análisis}

Tal como se ha comentado anteriormente, los estudiantes de turismo debían responder a diferentes cuestiones relativas al trabajo de gestión comercial, agrupadas en dos grandes áreas: el trabajo del vendedor y el comportamiento de los vendedores. Para llevar a cabo el análisis estadístico se utilizó el paquete estadístico SPSS 22.0, utilizando el análisis descriptivo y el análisis de las diferencias de medias (ANOVA) para responder a las cuestiones a investigar. Adicionalmente se recurrió al Análisis Factorial de Componentes Principales con la finalidad de confirmar los constructos analizados, para analizar la fiabilidad de cada una de estas escalas también se ha calculado su correspondiente $\alpha$-Cronbach. Con el objetivo de clasificar a los estudiantes en función de su futuro profesional en actividades de tipo comercial se realizó un análisis cluster o de conglomerados jerárquicos.

Previo a la obtención de resultados, se evaluaron las características psicométricas de cada una de las escalas empleadas en el estudio, para ello fue necesaria la realización de diversos análisis factoriales confirmatorios de primer orden. En este sentido, se llevaron a cabo diferentes fases que permitiesen dicha evaluación. En una primera fase se calcularon las correlaciones ítem-total de forma aislada para cada escala utilizada en el cuestionario. Siguiendo las recomendaciones de Saxe y Weitz (1982), todos los ítems debían presentar un valor superior a 0,35, para posteriormente, tal como indican Anderson y Gerbing (1988), llevar a cabo el análisis factorial confirmatorio. Seguidamente, tras depurar las 
escalas, se calculó la consistencia interna de cada una a través de los índices propuestos por Fornell y Larcker (1981) y Bagozzi y Yi (1988), el índice de la varianza extraída y la fiabilidad compuesta respectivamente. Por último, se comprobó la validez de constructo (convergente y discriminante) de las escalas. Para la realización de los análisis factoriales confirmatorios se recurrió a el paquete estadístico EQS, empleando el método de máxima verosimilitud robusto para evitar problemas con los datos (Hair, Anderson, Tatham y Black, 1999).

\section{RESULTADOS}

Para responder a las cuestiones planteadas anteriormente se ha procedido a realizar diferentes análisis. En primer lugar, se han clasificado a los estudiantes universitarios de turismo en función de su interés en las actividades comerciales para, posteriormente, analizar sus valoraciones sobre este tipo de trabajo. Los diferentes resultados se mostrarán para el total de la muestra y para cada uno de los grupos resultantes.

En segundo lugar, y para poder conocer la opinión de los estudiantes universitarios de turismo sobre la realización de trabajos de tipo comercial, seguimos la propuesta de Bristow et al. (2006) en su análisis sobre la opinión de los universitarios sobre el trabajo de los vendedores. Este constructo fue desglosado en tres factores: visión sobre la venta como futura actividad laboral (6 ítems), cómo creen que otras personas valoran la figura del vendedor (4 ítems) y, por último, opinión sobre la orientación al cliente de los vendedores (6 ítems). Para confirmar la existencia de estos factores se realizó un análisis factorial previo, cuyo resultado confirmó la existencia de los factores propuestos inicialmente. Se empleó la rotación Varimax, con un índice KMO de 0,804 y explicando los tres factores resultantes el $59,77 \%$ de la varianza total.

Además, deseábamos conocer la opinión de los estudiantes universitarios de turismo en relación a cuestiones éticas de los vendedores. Para ello, seguimos la propuesta de Dabholkar y Kellaris (1992), denominada "Personal Selling Ethics" (PSE), y utilizada posteriormente por diferentes autores (Stevenson y Bodkin, 1998; Donoho et al., 1999; Sundar y Kellaris, 2016; Sulsky, Marcus y McDonald, 2016; entre otros). Por ello, se les pedía a los encuestados que indicaran si eran aceptables, o no, una serie de 20 afirmaciones respecto a posibles acciones de los vendedores. Por ejemplo, realizar falsas promesas para poder cerrar una venta o excederse en los gastos de representación. Estas afirmaciones están redactadas de forma que a menor puntuación, mayor rechazo al comportamiento no-ético propuesto en la misma.

\subsection{Grado de interés en la actividad comercial}

Un primer objetivo es conocer la opinión de los estudiantes universitarios de turismo sobre su futuro profesional, y conocer si su opinión cambia en función del interés por desarrollar su actividad profesional en actividades de tipo comercial. Se realizó un análisis cluster utilizando la información sobre su interés en este tipo de trabajo y la probabilidad que asignaban a trabajar en este tipo de tareas. Este análisis nos permite obtener un conjunto de grupos de estudiantes que comparten características similares entre sí y diferentes 
al resto de grupos. Estos grupos deberán maximizar su homogeneidad interna (intragrupo) y, al mismo tiempo, ser diferentes al resto de grupos, es decir, maximizar su heterogeneidad con el resto de grupos. El método utilizado ha sido el método de Ward, dentro de los denominados de varianza, y agrupa los individuos de forma jerárquica de modo que minimiza la variación intra-grupal de la estructura formada (Mínguez y Fuentes, 2004). Para determinar el número adecuado de grupos, o clusters, analizamos el dendograma y se determinó que el número de grupos adecuado era de tres.

En la Tabla 1, se muestran los resultados del análisis de las diferencias de las medias (ANOVA) entre los tres grupos, todas ellas son significativas, y el resultado del total de la muestra. Previamente hemos comprobado que se cumplen las condiciones de aplicabilidad requeridas. Este procedimiento se ha seguido en los diferentes análisis realizados en el trabajo.

En general podemos indicar que los estudiantes universitarios de turismo no muestran un gran interés por trabajar en actividades comerciales, aunque son bastante conscientes de que es probable que deban realizar este tipo de tareas profesionales en el futuro.

El grupo 1, que denominaremos "Reacios", son el 12,7\% de los encuestados. Este grupo está formado por alumnos que no manifiestan interés en trabajar en actividades comerciales, y no consideran probable trabajar en esta actividad profesional.

El grupo 2, integrado por el $40,5 \%$ de la muestra, son aquellos alumnos que otorgan valores intermedios a ambas cuestiones, los denominaremos "Indecisos".

Por último, el grupo 3, los "Proclives", lo forman el 46,8\% de los encuestados. Este tercer grupo está formado por aquellos alumnos que están interesados en seguir su carrera profesional en el área comercial y que también consideran muy probable el tener que hacerlo.

Tabla 1

INTERÉS POR LA ACTIVIDAD COMERCIAL

\begin{tabular}{|l|c|c|c|c|}
\cline { 2 - 5 } \multicolumn{1}{c|}{} & TOTAL & Reacios & Indecisos & Proclives \\
\hline $\begin{array}{l}\text { ¿Qué interés tienes en seguir una carrera } \\
\text { en ventas tras acabar tus estudios? }\end{array}$ & 2,67 & $1,60^{*}$ & $2,34^{*}$ & $3,23^{*}$ \\
\hline $\begin{array}{l}\text { Tras acabar tus estudios, ¿Qué } \\
\text { probabilidad hay de que durante un } \\
\text { tiempo trabajes como vendedor? }\end{array}$ & 3,03 & $2,26^{*}$ & $2,86^{*}$ & $3,40^{*}$ \\
\hline
\end{tabular}

Escala: 1 "Nada" hasta 5"Mucho"

* Diferencias significativas entre los grupos, $\mathrm{p}<0.05$

\subsection{Valoración de la actividad comercial como profesión}

Para conocer la opinión que tienen los estudiantes respecto a la actividad comercial como carrera profesional, tal como se ha indicado, seguimos la propuesta de Bristow et al. (2006) y utilizamos una escala tipo Likert desde 1= "Muy en desacuerdo" hasta 5= "Muy de acuerdo" para responder a los 6 ítems planteados. 
Como puede observarse (Tabla 2), el conjunto total de los estudiantes analizados expresa una opinión positiva respecto de las tareas comerciales como futura actividad profesional. Los 6 ítems están por encima del 3,30 en una escala de 1 a 5. Podemos resaltar la idea de que las ventas pueden ser una buena oportunidad profesional, pudiendo ser incluso una profesión divertida, siendo la media global del constructo de 3,45.

Respecto a las diferencias entre los tres grupos, son significativas en todos los casos. Se observa que aquellos alumnos de turismo que muestran un mayor interés en seguir una carrera profesional en el ámbito comercial (Proclives) presentan valores más altos que los otros dos. En este grupo prácticamente todos los valores se encuentran próximos al 4.

Por su parte, los alumnos con escaso interés en las tareas comerciales (Reacios) no ven esta salida profesional como una alternativa interesante desde el punto de vista personal. No obstante, sí creen que puedes ser una oportunidad profesional de futuro.

El grupo de "Indecisos", valora de forma positiva las futuras oportunidades de una carrera profesional en el área comercial, pero su valoración personal de esta actividad es poco positiva (aunque no es negativa).

Tabla 2

VALORACIÓN DE LA ACTIVIDAD COMERCIAL

\begin{tabular}{|l|c|c|c|c|}
\cline { 2 - 5 } \multicolumn{1}{c|}{} & TOTAL & Reacios & Indecisos & Proclives \\
\hline $\begin{array}{c}\text { Valoración global de la actividad } \\
\text { comercial }(\alpha=0,861)\end{array}$ & $\mathbf{3 , 4 5}$ & $\mathbf{2 , 2 3 *}$ & $\mathbf{3 , 1 8 *}$ & $\mathbf{4 , 0 2 *}$ \\
\hline $\begin{array}{l}\text { Puede ofrecerme oportunidades } \\
\text { profesionales }\end{array}$ & 3,68 & $2,91^{*}$ & $3,45^{*}$ & $4,08^{*}$ \\
\hline $\begin{array}{l}\text { Puede ofrecerme oportunidades de } \\
\text { crecimiento }\end{array}$ & 3,55 & $2,91 *$ & $3,29 *$ & $3,95^{*}$ \\
\hline Puede ser divertida y excitante & 3,40 & $1,73^{*}$ & $3,24 *$ & $3,98^{*}$ \\
\hline Puede ser satisfactoria & 3,39 & $1,98^{*}$ & $3,05^{*}$ & $4,05^{*}$ \\
\hline Puede ser gratificante & 3,38 & $2,05^{*}$ & $3,04^{*}$ & $4,03^{*}$ \\
\hline $\begin{array}{l}\text { Disfrutaría con los desafíos del } \\
\text { trabajo }\end{array}$ & 3,32 & $1,80^{*}$ & $2,98^{*}$ & $4,02^{*}$ \\
\hline
\end{tabular}

Escala: 1 "Muy en desacuerdo" hasta 5"Muy de acuerdo"

* Diferencias significativas entre los grupos, $\mathrm{p}<0.05$

Estas diferencias nos permiten indicar que aquellos alumnos que perciben y aceptan en mayor medida un futuro relacionado con las actividades comerciales consideran que estas funciones les ofrecerán una carrera profesional satisfactoria personal y laboralmente.

\section{3. ¿Cómo ven otros a los vendedores?}

Otra de las cuestiones analizadas respecto de las actividades comerciales, ha sido la relativa a la imagen que la sociedad tiene de esta profesión. En concreto, se preguntaba a los estudiantes universitarios de turismo sobre cómo creían ellos que otras personas veían a los comerciales en el sector turístico (Bristow et al., 2006). 
Los resultados globales obtenidos (Tabla 3) reflejan que, según los encuestados, la figura del comercial no es demasiado positiva. El valor medio es de 2,69 (sobre 5), no superando el 3 en ningún caso. Esta puede ser una explicación al relativo rechazo de los estudiantes universitarios de turismo a desarrollar su futuro profesional en el área comercial.

A priori, pensábamos que aquellos estudiantes que tenían interés en el área comercial (Proclives, y en menor medida Indecisos) considerarían que la valoración social del trabajo comercial es más positiva que los del grupo "Reacios", puesto que mostraron interés en que ese sea su ámbito profesional futuro.

Los valores medios del grupo "Proclives" muestran una mejor valoración de la imagen social de los comerciales que los de los otros dos grupos. Los alumnos más interesados (Proclives), pese a que no creen que la imagen social de los comerciales sea demasiado positiva, consideran que los vendedores son mejor vistos y aceptados que los integrantes del grupo "Reacios". No obstante, no existen suficientes diferencias estadísticamente significativas para afirmarlo.

Tabla 3

IMAGEN SOCIAL DE LOS VENDEDORES

\begin{tabular}{|l|c|c|c|c|}
\cline { 2 - 5 } \multicolumn{1}{c|}{} & TOTAL & Reacios & Indecisos & Proclives \\
\hline $\begin{array}{l}\text { Imagen global de los vendedores } \\
(\alpha=0,750)\end{array}$ & $\mathbf{2 , 6 9}$ & $\mathbf{2 , 4 9 *}$ & $\mathbf{2 , 6 5}$ & $\mathbf{2 , 7 9 *}$ \\
\hline $\begin{array}{l}\text { Muchos vendedores son honestos } \\
\text { y sinceros }\end{array}$ & 2,96 & 2,89 & 2,84 & 3,09 \\
$\begin{array}{l}\text { Muchos vendedores son aceptados } \\
\text { positivamente }\end{array}$ & 2,90 & $2,68 *$ & 2,89 & $2,98^{*}$ \\
$\begin{array}{l}\text { La gente tienen en alta } \\
\text { consideración a los vendedores } \\
\text { Los vendedores son admirados por } \\
\text { la gente }\end{array}$ & 2,46 & 2,23 & 2,49 & 2,49 \\
\hline
\end{tabular}

Escala: 1 "Muy en desacuerdo" hasta 5"Muy de acuerdo"

* Diferencias significativas entre los grupos, $\mathrm{p}<0.05$

\subsection{Orientación al cliente de los vendedores}

La valoración que los estudiantes universitarios de turismo realizan del enfoque u orientación al cliente de los vendedores se midió con una serie de afirmaciones de sentido inverso (Bristow et al., 2006); de modo que a mayor puntuación (5= "Muy de acuerdo") menor orientación al cliente. El objetivo era conocer cómo ven los estudiantes la preocupación de los vendedores hacia la satisfacción de las necesidades y deseos del cliente frente al logro de los objetivos puramente personales de los mismos.

Las conclusiones respecto a este objetivo son poco positivas, al menos desde el punto de vista de la gestión comercial. En general, podemos decir que los encuestados no creen 
que los vendedores estén orientados al cliente, pues todos los resultados están por encima del 3,5 en una escala de 1 a 5 (Tabla 4).

Tabla 4

ORIENTACIÓN AL CLIENTE DEL VENDEDOR

\begin{tabular}{|l|c|c|c|c|}
\cline { 2 - 5 } \multicolumn{1}{c|}{} & TOTAL & Reacios & Indecisos & Proclives \\
\hline $\begin{array}{l}\text { Orientación global al cliente } \\
(\alpha=0,702)\end{array}$ & $\mathbf{3 , 6 8}$ & $\mathbf{3 , 7 6}$ & $\mathbf{3 , 6 6}$ & $\mathbf{3 , 6 8}$ \\
\hline $\begin{array}{l}\text { Exageran las ventajas de sus } \\
\text { productos }\end{array}$ & 4,04 & 4,07 & 4,03 & 4,04 \\
\hline $\begin{array}{l}\text { Ocultan la verdad para lograr } \\
\text { una venta }\end{array}$ & 3,82 & 3,89 & 3,79 & 3,82 \\
\hline $\begin{array}{l}\text { Se aprovechan de los clientes } \\
\text { con poca formación }\end{array}$ & 3,71 & 3,80 & 3,70 & 3,71 \\
\hline $\begin{array}{l}\text { Venden productos que la gente } \\
\text { no necesita }\end{array}$ & 3,59 & 3,86 & 3,55 & 3,55 \\
\hline $\begin{array}{l}\text { Falsifican garantías, dan falsas } \\
\text { promesas }\end{array}$ & 3,50 & 3,34 & 3,47 & 3,57 \\
\hline $\begin{array}{l}\text { No admiten que no saben } \\
\text { responder a las dudas }\end{array}$ & 3,43 & 3,61 & 3,40 & 3,40 \\
\hline
\end{tabular}

Escala: 1 "Muy en desacuerdo" hasta 5"Muy de acuerdo"

* Diferencias significativas entre los grupos, $\mathrm{p}<0.05$

Además, no existen diferencias significativas relacionadas con el interés por seguir una carrera profesional en ventas. Este resultado es coherente con la imagen que, según los estudiantes, tiene la sociedad del trabajo de vendedor.

\subsection{Valoración del comportamiento ético de los vendedores}

Para conocer la opinión de los estudiantes universitarios de turismo sobre el comportamiento ético de los vendedores, seguimos la propuesta de Dabholkar y Kellaris (1992), denominada "Personal Selling Ethics" (PSE).

En primer lugar realizamos un análisis factorial con las 20 afirmaciones de la escala PSE (Dabholkar y Kellaris, 1992). Se trata de una escala inversa, de modo que a valores más bajos, el comportamiento descrito es considerado como menos ético por parte de los encuestados. Se obtuvieron cinco factores (KMO de 0,904 y varianza total explicada igual a $61,85 \%$ ):

- Primer factor, denominado "Comportamiento con clientes", está formado por 6 ítems, que recogen acciones que pueden desarrollar los vendedores durante sus 
interacciones con los clientes y que pueden traducirse en comportamientos no éticos.

- Segundo factor, "Gestión de recursos", lo forman 5 ítems relativos al uso de diferentes tipos de recursos, económicos o de otro tipo, que las empresas ponen a disposición de los vendedores para realizar su trabajo, y que estos pueden destinar a cuestiones distintas a aquellas paras las que fueron creadas.

- Tercer factor "Acciones no legales", lo forman 3 ítems, son acciones que podrían llegar a suponer problemas legales tanto para el vendedor como para su empresa.

- Cuarto factor, "Comportamiento con la empresa", lo forman 3 ítems, se refiere a comportamientos relacionados con la confianza entre el vendedor respecto a la empresa para la que trabaja.

- Quinto factor, "Presión comercial”, lo forman 3 ítems, y se refieren a posibles actos para presionar a los clientes en sus relaciones comerciales.

En general, el análisis de los resultados obtenidos (Tabla 5) muestra que los estudiantes valoran muy negativamente, inaceptable, el comportamiento no ético de los vendedores en el desarrollo de su actividad profesional. En general todos los ítems se encuentran por debajo de 2,5 en una escala de 1 a 5 puntos.

Respecto a las valoraciones de los diferentes factores, podemos indicar que los comportamientos no éticos menos aceptables se refieren a los que los vendedores realizan durante la interacción directa con sus clientes (media global de 1,62) y en relación a su propia empresa (media de 1,97). También son poco aceptables la realización de acciones que pueden considerarse como ilegales (media de 2,01) y el mal uso de los recursos de que disponen los vendedores (media de 2,08). En sentido contrario, se considera casi aceptable presionar a los clientes para alcanzar los objetivos comerciales (media de 2,97).

Tabla 5

COMPORTAMIENTO ÉTICO DE LOS VENDEDORES

\begin{tabular}{|l|c|c|c|c|}
\cline { 2 - 5 } \multicolumn{1}{c|}{} & TOTAL & Reacios & Indecisos & Proclives \\
\hline $\begin{array}{l}\text { Comportamiento con clientes } \\
(\alpha=0,857)\end{array}$ & $\mathbf{1 , 6 2}$ & $\mathbf{1 , 6 5}$ & $\mathbf{1 , 6 7}$ & $\mathbf{1 , 5 7}$ \\
\hline $\begin{array}{l}\text { Incrementar los precios } \\
\text { indebidamente }\end{array}$ & 1,83 & 1,75 & 1,91 & 1,79 \\
\hline $\begin{array}{l}\text { Hacer trampas en los concursos } \\
\text { de venta }\end{array}$ & 1,77 & 1,84 & 1,84 & 1,69 \\
\hline $\begin{array}{l}\text { Realizar falsas promesas para } \\
\text { cerrar ventas }\end{array}$ & 1,77 & 1,75 & 1,81 & 1,74 \\
\hline $\begin{array}{l}\text { Hacer trampas en un proceso de } \\
\text { subasta }\end{array}$ & 1,61 & 1,77 & 1,59 & 1,58 \\
\hline Pinchar el teléfono & 1,43 & 1,50 & 1,52 & 1,34 \\
\hline Usar amenazas para cerrar ventas & 1,30 & 1,30 & 1,33 & 1,27 \\
\hline
\end{tabular}




\begin{tabular}{|c|c|c|c|c|}
\hline & \\
\hline & TOTAL & Reacios & Indecisos & Proclives \\
\hline Gestión de recursos $(\alpha=0,792)$ & 2,08 & 2,11 & 2,09 & 2,05 \\
\hline $\begin{array}{l}\text { Conflicto de intereses con la } \\
\text { empresa }\end{array}$ & 2,42 & 2,59 & 2,43 & 2,36 \\
\hline $\begin{array}{l}\text { Excederse en los gastos de } \\
\text { representación }\end{array}$ & 2,33 & 2,27 & 2,42 & 2,26 \\
\hline Mal uso de la jornada laboral & 2,01 & 2,11 & 2,00 & 1,98 \\
\hline $\begin{array}{l}\text { Irse de vacaciones en horario } \\
\text { laboral }\end{array}$ & 1,82 & 1,86 & 1,79 & 1,83 \\
\hline \multirow[t]{2}{*}{ Inflar los informes de gastos } & 1,80 & 1,70 & 1,81 & 1,82 \\
\hline & TOTAL & Reacios & Indecisos & Proclives \\
\hline Acciones no legales $(\alpha=0,722)$ & 2,01 & 2,19 & 1,92 & 2,05 \\
\hline $\begin{array}{l}\text { Copiar de la competencia en una } \\
\text { feria }\end{array}$ & 2,25 & 2,48 & 2,11 & 2,32 \\
\hline Ofrecer sobornos en especie & 2,05 & 2,16 & 1,99 & 2,07 \\
\hline \multirow[t]{2}{*}{$\begin{array}{l}\text { Ofrecer un soborno económico a } \\
\text { un cliente }\end{array}$} & 1,74 & 1.93 & 1,66 & 1,76 \\
\hline & TOTAL & Reacios & Indecisos & Proclives \\
\hline $\begin{array}{c}\text { Comportamiento con la } \\
\text { empresa }(\alpha=0,710)\end{array}$ & 1,97 & 2,24 & 2,03 & 1,83 \\
\hline $\begin{array}{l}\text { Inflar el currículum, mentir a la } \\
\text { empresa }\end{array}$ & 2,23 & 2,41 & 2,31 & 2,10 \\
\hline Dejar la empresa sin aviso previo & 2,03 & 2,3 & 2,08 & 1,91 \\
\hline \multirow[t]{2}{*}{$\begin{array}{l}\text { Pasar información a la } \\
\text { competencia }\end{array}$} & 1,65 & 2,00 & 1,71 & 1,49 \\
\hline & TOTAL & Reacios & Indecisos & Proclives \\
\hline Presión comercial $(\alpha=0,682)$ & 2,97 & 2,98 & 2,94 & 2,99 \\
\hline Espiar a la competencia & 3,26 & 3,36 & 3,19 & 3,28 \\
\hline Uso de trucos para cerrar ventas & 3,14 & 3,07 & 3,12 & 3,19 \\
\hline $\begin{array}{l}\text { Obligar al cliente a quedarse con } \\
\text { muestras }\end{array}$ & 2,50 & 2,52 & 2,50 & 2,51 \\
\hline
\end{tabular}

Escala: 1 "Totalmente inaceptable" hasta 5"Totalmente aceptable"

$*$ Diferencias significativas entre los grupos, $p<0.05$

En la mayoría de los factores del comportamiento ético, es el grupo de los menos interesados (Reacios) el que considera menos inaceptable este tipo de comportamiento no ético. No obstante, al analizar los resultados para los tres grupos descritos, no aparecen diferencias significativas en ninguno de los ítems analizados. 


\section{DISCUSIÓN Y CONCLUSIONES}

En la presente investigación hemos analizado la opinión que los estudiantes universitarios de turismo tienen respecto al trabajo desarrollado por los gestores comerciales, o vendedores, así como su valoración respecto a tener un futuro profesional en el sector comercial turístico. En concreto, les hemos preguntado sobre su interés por desarrollar su carrera profesional en actividades comerciales en empresas turísticas, su opinión y el de la sociedad sobre la figura de los vendedores y cómo consideran que se comportan estos profesionales en el desarrollo de sus actividades.

En primer lugar, podemos clasificar a los estudiantes en tres grupos diferenciados en función a grado de interés por trabajar en tareas comerciales, y la probabilidad que asignan a tener que hacerlo. Un primer grupo no tiene interés en hacerlo, otro grupo podría ser calificado como indiferente o indeciso y un tercer grupo que muestran cierto grado de interés. En general, no parece que a estos estudiantes les genere mucho interés trabajar como comerciales, o vendedores, pese a que es una de las actividades profesionales más demandas por las empresas (Informe Infojobs, 2018).

No obstante, hay una mayoría que son conscientes de que su futuro profesional es probable que se desarrolle en las actividades comerciales. Desde el punto de vista de la formación, y dados los niveles de desempleo o de empleos de nivel inferior a la formación de los candidatos a ocuparlos, parece adecuado incorporar en los estudios universitarios de turismo algún tipo de formación relacionada con el trabajo de los vendedores en el sector turístico, y no únicamente con formación de marketing. Esta formación facilitará el logro de los puestos de trabajo en el entorno comercial y hará conscientes a los alumnos de turismo de la importancia cuantitativa y cualitativa de esta actividad profesional en el sector turístico. Además, el incremento de la formación mejora también el salario de los trabajadores del sector turístico (Ons-Cappa, García-Pozo y Sánchez-Ollero, 2017).

Con relación a la valoración de la actividad comercial, es el grupo de los más interesados el que mejor valora los diferentes aspectos que el trabajo comercial les puede aportar profesional y personalmente, les parece una buena oportunidad profesional y un trabajo gratificante e incluso divertido. Una opinión contraria es la que indican aquellos estudiantes que no consideran el trabajo comercial como parte de su futuro, y como mucho lo valoran como oportunidad de mejora profesional.

Dado que un contacto en las aulas con asignaturas relacionadas con el trabajo comercial en empresas turísticas hará que mejore la actitud de los estudiantes hacia este tipo de trabajo (Peltier et al., 2014), es recomendable que el contenido de estas permitiese a los alumnos entender de modo adecuado el trabajo de un vendedor profesional. Los vendedores en la actualidad son uno de los pilares básicos del éxito de las empresas turísticas, por ello deben saber trabajar en equipo, utilizar los diferentes recursos tecnológicos disponibles y saber centrarse en conocer y satisfacer las necesidades y demandas de los clientes; esto implica que es una actividad creativa, innovadora y posiblemente atractiva para los estudiantes universitarios de turismo.

Respecto a la imagen social de los vendedores, en opinión de los estudiantes, no podemos calificarla como muy positiva, y es bastante similar entre los tres grupos identificados. Esta imagen, habitual en los estudios similares realizados en diferentes contextos, dificulta 
la tarea de los responsables en la selección de comerciales pues puede frenar a potenciales candidatos a cubrir esos puestos, y al mismo tiempo hace que algunos estudiantes se autolimiten profesionalmente.

Esta visión puede modificarse cuando los alumnos sean conscientes de la importancia del vendedor en la toma de decisiones de los clientes, tanto si se trata de venta entre empresas (por ejemplo, los comerciales de un touroperador) como si se trata de la venta a clientes finales, turistas (por ejemplo, la venta en una agencia de viajes). Por ello, los vendedores deben ser capaces de generar expectativas y emociones en el cliente potencial para que éste tome la decisión de comprar, deben lograr que la compra-venta se convierta en una experiencia positiva. Además, la formación en ventas afecta al comportamiento de los futuros comerciales con la empresa, de forma que será más fácil la integración y el compromiso con la misma.

Otra de las cuestiones analizadas ha sido la valoración sobre el comportamiento de los vendedores en el desarrollo de sus tareas. En primer lugar, hemos analizado el grado de orientación al cliente por parte de los vendedores, y las conclusiones en este caso tampoco son muy positivas. Todos los grupos consideran que, en general, los vendedores se preocupan más por alcanzar sus propios objetivos que por ayudar a satisfacer las necesidades y deseos de sus clientes. En segundo lugar, se ha pedido a los estudiantes que valoran posibles comportamientos no éticos de los vendedores. Podemos indicar que los tres grupos de estudiantes consideran que no es aceptable este tipo de comportamiento por parte de los vendedores, especialmente en su relación con los clientes y la empresa. No obstante, las acciones que pueden suponer algún tipo de presión para facilitar el logro de los objetivos son admitidas por todos ellos.

Por ello, y dado que el entorno del trabajo comercial puede provocar comportamientos no adecuados y facilitar el desempeño de acciones no éticas, es importante y adecuado la inclusión en la formación universitaria de contenidos relativos a la importancia del análisis ético en todas sus decisiones. Por ejemplo, incluyendo sesiones de role-playing o simulación, que permiten a los estudiantes situarse en un entorno cercano a la realidad profesional de la venta.

Como toda investigación, este trabajo tiene ciertas limitaciones, centradas fundamentalmente en la muestra analizada. Los estudiantes encuestados cursan sus estudios en una única universidad, aunque en diferentes cursos; sería adecuado repetir el estudio en otras universidades a fin de poder generalizar los resultados. También se plantea como futura investigación, la comparación de los resultados con los de estudiantes de otras áreas relacionadas, por ejemplo las relacionadas con la gestión empresarial, u otras diferentes.

\section{BIBLIOGRAFÍA}

AGNIHOTRIL, R., BONNEY, L. DIXON, A. ERFFMEYER, R. PULLINS, E. SOJKA, J. y WEST, V. (2014): "Developing a Stakeholder Approach for Recruiting Top-Level Sales Students”, Journal of Marketing Education, vol. 36 (1), pp. 75-86.

AMIN, S. G., HAYAJNEH, A.F., y NWAKANMA, H. (1995): “College students' views of sales jobs as a career: An empirical investigation”. American Business Review, vol. 13 , pp. 54-61. 
ANECA (2004): Libro Blanco del Grado en Turismo. Madrid.

BABIN, B., BOLES, J. y ROBIN, D. (2000): "representing the perceived ethical work climate among marketing employees," Academy of Marketing Science, 28 (3), pp. 345-358.

BAGOZZI, R.P., BELSCHAK, F. y VERBEKE, W. (2010): “The role of emotional wisdom in salesperson's relationships with colleagues and customers", Psychology and Marketing, vol. 27 (11), pp. 1001-1031.

BAGOZZI, R.P. y YI, Y. (1988): “On the evaluation of structural equation models.” Journal of the Academy of Marketing Science, vol. 16, pp. 74-94.

BAHHOUTH, V., SPILLAN, J.E. y JÓZEFOWSKI, B. (2011): "Predictive power of personal factors in studying students' perception of sales profession in Poland", Journal of East-West Business, vol. 17 (4), pp. 195-212.

BAHHOUTH, V., SPILLAN, J. y KARSAKLIAN, E. (2014): “Are students driven by negative or positive perception about sales profession in France?" European Journal of Business and Social Sciences, vol. 3 (1), pp 16-32.

BAHHOUTH, V., SPILLAN, J. y MENSAH, E. (2013) "Predictive power of personal factors in studying students' perception of sales profession in Ghana", International Journal of Business and Social Science, vol. 4 (7), pp. 291-296.

BAKKER, A. y DEMEROUTI, E. (2007): "The job demands-resources model: state of the art", Journal of Managerial Psychology, vol. 22 (3), pp. 309-328.

BALLESTRA, L- V., CARDINALI, S. y PALANGA, P. (2017): "The changing role of salespeople and unchanging feeling toward selling: Implications for the HEI programs". Journal of Marketing Education, vol. 39 (3), pp. 176-189.

BARAT, S. y SPILLAN, J.E. (2009): “A cross country comparative analysis of students' perceptions of the sales profession: A look at U.S., Peru, and Guatemala". Marketing Management Journal, vol. 19 (2), pp. 52-63.

BLOCKER, C.P., CANNON, J., PANAGOPOUlOS, N.G. y SAGER, J.K. (2012): "The role of the sales force in value creation and appropriation: New directions for research". Journal of Personal Selling \& Sales Management, vol. 32, pp. 15-27.

BOLANDER,W., BONNEY, L. y SATORNINO, C. (2014), "Sales education efficacy: examining the relationship between sales education and sales success", Journal of Marketing Education, vol. 36 (2), pp. 169-181.

BRISTOW, D.N., AMYX, D., CASTLEBERRY, S.B. y COCHRAN, J.J. (2011): “A cross-generational comparison of motivational factors in a sales career among Gen-X and Gen-Y college Students", Journal of Personal Selling \& Sales Management, vol. 31 (1), pp. 77-85.

BRISTOW, D.N., AMYX, D. y SLACK, J. (2006), “An empirical look at professional selling from a student perspective", Journal of Education for Business, vol. 81 (5), pp. 242-249.

BRISTOW, D.N. y GULATI, R. (2002), "The teaching of sales related courses at the university level: an empirical look from the sales manager's perspective", Journal of Selling and Major Account Management, vol. 4 (3), pp. 25-43. 
BURNETT, M., PETTIJOHN, C. y KEITH, N. (2008). "A comparison of the ethical perceptions of prospective personal selling and advertising employees". Marketing Management Journal, vol. 18 (1), pp. 77-83.

CABALLERO, R. y JOONAS, K. (2009), "Mexican students' attitudes towards personal selling: an exploratory investigation", AIMS International, vol. 3 (3), pp. 221-240.

CABALLERO, C. y WALKER, A. (2010): "Work readiness in graduate recruitment and selection: A review of current assessment methods". Journal of Teaching and Learning for Graduate Employability, vol. 1, pp. 13-25.

CANALES, P. (2012): "La imagen del vendedor profesional entre los universitarios". 3cEmpresa, vol. 4, pp. 6-16.

CONCHA, A., KUMAR, P., TARASIL, C. y WILSON, H. (2014), "Selling sales factors influencing undergraduate business students' decision to pursue sales education", Journal of Marketing Education, vol. 36 (2), pp. 94 -104.

CRAVENS,D.W., INGRAM, T.N., LAFORGE, R.W. y YOUNG, C.E. (1993): “BehaviorBased and Outcome-Based salesforce control systems". Journal of Marketing, vol. 57, pp. 47-59.

CRON, W.L., BALDAUF, A., LEIGH, T.W. y GROSSENBACHER, S. (2014): "The strategic role of the sales force: Perceptions of senior sales executives". Journal of the Academy of Marketing Science, vol. 42, pp. 471-489.

CROSS, M., BRASHEAR, T., RIGDON, E. y BELLENGER, D.N. (2007): “Customer orientation and salesperson performance", European Journal of Marketing, vol. 41, (7/8), pp. 821-835.

CUMMINS, S.W., PELTIER, J.W., POMIRLEANU, N., CROSS, J. y SIMON, R. (2015): "Evaluating educational practices for positively affecting student perceptions of a sales career", Journal of Marketing Education, vol. 37 (1), pp. 25-35.

DABHOLKAR, O. y KELLARIS, J. (1992). “Toward understanding marketing students' ethical judgment of controversial personal selling practices". Journal of Business Research, Vol. 24, pp. 313-329.

DUBINSKY, A.J. (1980): "Recruiting college students for the salesforce". Industrial Marketing Management, vol. 9, pp. 37-45.

EXCELTUR (2006): Libro blanco de los Recursos Humanos en España. Madrid.

FORNELL, C. y LARCKER, D.F. (1981): "Evaluating structural models with unobservable variables and measurement error". Journal of Marketing Research, vol. 28, pp. 39-50.

HAIR, J.F., ANDERSON, R.E., TATHAM, R.L. y BLACK, W.C. (1999): Análisis multivariante, $5^{\mathrm{a}}$ edición, Prentice-Hall. Madrid.

HARTMAN, K.B. (2006): "Television and movie representations of salespeople: beyond Willie Loman”, Journal of Personal Selling and Sales Management, vol. 26 (3), pp. 293-292.

HAWES, J.M., RICH, A.K. y WIDMIER, S.M. (2004), “Assessing the development of the sales profession", Journal of Personal Selling and Sales Management, vol. 24 (1), pp. 27- 37. 
HONEYCUTT, E. D., JR., FORD, J. B., SWENSON, M.J. y SWINYARD, W.R. (1999): "Student preferences for sales careers around the Pacific Rim". Industrial Marketing Management, vol. 28, pp. 27-36.

INGRAM, T.N., LAFORGE, R.W. y LEIGH, T.W. (2002): "Selling in the new millennium: A joint agenda". Industrial Marketing Management, vol. 31, pp. 559-567.

KAVAS, A. (2003): “African-American students' attitudes toward personal selling as a career”. Negro Educational Review, vol. 54(1-2), pp. 31-38.

KERIN, R.A., HARTLEY, S.W. y RUDELIUS, W. (2009): Marketing (9th ed.), McGrawHill Irwin, Boston, MA.

KESZEY, T. y BIEMANS, W. (2017): “Trust in marketing's use of information from sales: the moderating role of power". Journal of Business \& Industrial Marketing, vol. 32 (2), pp. 258-273.

KLEIN, T. A., LACZNIAK, G.R. y MURPHY, P.E. (2006): "Ethical marketing: A look on the bright side". Marketing Management Journal, vol. 16 (1), pp. 228-243.

LASSK, F.G., INGRAM, T.N., KRAUS, F. y DIMASCIO, R. (2012), “The future of sales training: challenges and related research questions", Journal of Personal Selling and Sales Management, vol. 32 (1), pp. 141-154.

LEE, N., BEATSON, A., GARRETT, T.C., LINGS, I. y ZHANG X. (2009), “A study of the attitudes towards unethical selling amongst Chinese salespeople", Journal of Business Ethics, vol. 88 (3), pp. 497-515

LEE, N., SANDFIELD, A. y DHALIWAL, B. (2007): “An empirical study of salesperson stereotypes amongst UK students and their implications for recruitment”. Journal of Marketing Management, vol. 23, pp. 723-744.

LILLO, A. (2009): "El papel del capital humano en el sector turístico: algunas reflexiones y propuestas". Cuadernos de Turismo, $\mathrm{n}^{\mathrm{O}} 24$, pp. 53-54.

LILLO, A., RAMÓN, A.B. y SEVILLA, M. (2007): "El capital humano como factor estratégico para la competitividad del sector turístico". Cuadernos de Turismo, no 19 , pp. 47-69.

LUTHY, M. (2007): "Influences on undergraduates considering a career in professional selling", Academy of Educational Leadership Journal, 11 (3), pp. 1-7.

MANNING, G. L., REECE, B. L. y AHEARNE, M. (2010). Selling today (11th ed.), Prentice Hall, Upper Saddle River, NJ.

MARCOS-CUEVAS, J., CRITTEN, P., SQUIRE, P. y SPEAKMAN, J.I.F. (2014), "Enhancing the professional mindset of future sales professionals: key insights from a master in sales transformation", Journal of Marketing Education, vol. 36 (2), pp. 144-155.

MARRERO, J.R. (2015): "La sobrecualificación en el sector turístico: el caso español (1987-2011)". Cuadernos de Relaciones Laborales, vol. 33 (1), pp. 149-168.

McCLAREN, N. (2000): "Ethics in personal selling and sales management: a review of the literature focusing on empirical findings and conceptual foundations", Journal of Business Ethics, 27 (3), pp. 285-303.

MÍNGUEZ, A. y FUENTES, M. (2004): Cómo hacer una investigación social, Tirant lo Blanch, Valencia. 
MOSSAZ, A. y COGHLAN, A. (2017): “The role of travel agents' ethical concerns when brokering information in the marketing and sale sustainable tourism", Journal of Sustainable tourism, vol. 25 (7), pp. 989-1006.

MUEHLING, D.D. y WEEKS, W. A. (1988): "Women's perceptions of personal selling: Some positive results". Journal of Personal Selling \& Sales Management, vol. 8 (1), pp. 11-20.

MULKI, J., JARAMILLO, F. y LOCANDER, W. (2009): "Effects of ethical climate and supervisory trust on salesperson's job attitudes and intentions to quit", Journal of Personal Selling \& Sales Management, 26 (1), pp. 19-26.

ONS-CAPPA, M., GARCÍA-POZO, A. y SÁNCHEZ-OLLERO, J.L. (2017): “Incidencia de factores personales y laborales en los salarios del sector hostelero: una visión de género". Cuadernos de Turismo, no 39, pp. 417-436.

OVIEDO-GARCIA, M.A. (2007), "Internal validation of a bio-data extraversion scale for salespeople", Social Behaviour and Personality: An International Journal, vol. 35 (5), pp. 675-692.

PAUL, J. y SHRIVASTAVA, A. (2015), “Comparing entrepreneurial communities: theory and evidence from a cross-country study in Asia", Journal of Enterprising Communities: People and Places in the Global Economy, vol. 9 (3), pp. 206-220.

PELTIER, J.W., CUMMINS, S., POMIRLEANU, N., CROSS, J. y SIMON, R. (2014): “A parsimonious instrument for predicting students' intent to pursue a sales career: Scale development and validation". Journal of Marketing Education, vol. 36, pp. 62-74.

PETTIJOHN, E.C., PETTIJOHN, S.L. y TAYLOR, J.A. (2007): “Does salesperson perception of the importance of sales skills improve sales performance, customer orientation, job satisfaction, and organizational commitment, and reduce turnover?" Journal of Personal Selling \& Sales Management, vol. 27(1), pp. 75-88.

PETTIJOHN, C.E. y PETTIJOHN, L.S. (2009): “An exploratory analysis of sales career desirability: An MBA perspective". Academy of Educational Leadership Journal, vol. 13(4), pp. 35-47.

RUIZ, J.I. (1996). Metodología de la investigación cualitativa. Bilbao: Universidad de Deusto.

SAXE, R. Y WEITZ, B.A. (1982): "The SOCO Scale: A Measure of the Customer Orientation of Salespeople". Journal of Marketing Research, vol. 19 , pp. 343-351.

RAMSEY, R., MARSHALL, G, JOHNSTON, M. y DEETER-SCHMELZ, D. (2007): "Ethical ideologies and older consumer perceptions of unethical sales tactics", Journal of Business Ethics, 70 (2), pp. 191-207.

SCHWEPKER, C.H. Jr. (2003): "Customer-oriented selling: a review, extension and directions for future research", Journal of Personal Selling \& Sales Management, vol. 23 (2), pp. 153-173.

SCHWEPKER, C.H., Jr. (2001): "Ethical climate's relationship to job satisfaction, organizational commitment and turnover in the sales force," Journal of Business Research, vol. 54 (1), 39-52.

SCHWEPKER, C.H. y GOOD, D (2004): "Marketing Control and Sales Force Customer Orientation", Journal of Personal Selling \& Sales Management, vol. 24 (3), pp. 167179. 
STEVENSON, T.H. y BODKIN, C.D. (1998): “A cross-national comparison of university students' perceptions regarding the ethics and acceptability of sales practices". Journal of Business Ethics, vol. 17, pp. 45-55.

STORBACKA, K., RYALS, L., DAVIES, I.A. y NENONEN, S. (2009): "The changing role of sales: Viewing sales as a strategic, cross-functional process". European Journal of Marketing, vol. 43, 890-906.

SULSKY, L., MARCUS, J. y McDONALD, H. (2016): "Examining ethicality judgements of theft behavior: the role of moral relativism". Journal of Business and Psychology, vol. 31 (3), pp. 383-398.

SUNDAR, A. y KELLARIS, J. (2016): "How logo colors influence shoppers' judgments of retailer ethicality: the mediating role of perceived eco-friendliness". Journal of Business Ethics, vol.4, pp. 1-17.

THOMAS, T., SCHERMERHORN, J.R. y DIENHART, J. (2004): “Strategic leadership of ethical behavior in business," Academy of Management Executive, vol. 18 (2), pp. 56-66.

VALENTINE, S. y BARNETT, T. (2003: "Ethics code awareness, perceived ethical values, and organizational commitment," Journal of Personal Selling \& Sales Management, vol. 23 (4), pp. 359-36.

WALDECK, N.E., PULLINS E.B. y HOULETTE, M. (2010), "Media as factor in student perceptions for sales jobs: a framework for research", Journal of Personal Selling \& Sales Management, vol. 30 (4), pp. 343-353.

WALKER, I., TSARENKO, Y., WAGSTAFF, P., POWELL, I., STEEL, M. y BRACEGOVAN, J. (2009), “The development of competent marketing professionals", Journal of Marketing Education, vol. 31 (3), pp. 253-263.

WEITZ, B. Y BRADFORD, K. (1999):'Personal selling and sales management: a relationship marketing perspective". Journal of the Academy of Marketing Science", vol. 27 (2), pp. 241-254.

WILES, M. A. y SPIRO, R.L. (2004): “Attracting graduates to sales position and the role of recruiter knowledge: A re-examination”. Journal of Personal Selling \& Sales Management, vol. 25, pp. 39-48.

WORTRUBA, T.R. (1996): "The transformation of industrial selling: causes and consequences”. Industrial Marketing Management, vol. 25 (5), pp. 327-338.

WRIGHT, R. y LUNDSTROM, W. (2004): "Physicians' perceptions of pharmaceutical sales representatives: a model for analyzing the customer relationship," International Journal of Medical Marketing, vol. 4 (1), pp. 29-38. 
